\title{
Inovações pedagógicas no panorama da COVID-19: um relato do projeto "Caixa
}

\author{
Mental" \\ Pedagogical innovations in the COVID-19 scenario: an report from the "Mind Box" project \\ Innovaciones pedagógicas en el panorama del COVID-19: un relato del proyecto "Caja Mental"
}

Recebido: 09/02/2021 | Revisado: 12/02/2021 | Aceito: 18/02/2021 | Publicado: 26/02/2021

Pablo Cleber Sousa Lopes Sales ORCID: https://orcid.org/0000-0003-2617-9398 Faculdade de Ciências Humanas, Exatas e da Saúde do Piauí, IESVAP, Brasil E-mail: pablo.clebersales@gmail.com

Almir Vieira de Sousa Neto

ORCID: https://orcid.org/0000-0002-9194-9232

Faculdade de Ciências Humanas, Exatas e da Saúde do Piauí, IESVAP, Brasil E-mail: vieira10101almir@gmail.com Lahuan Araujo Costa

ORCID: https://orcid.org/0000-0002-2490-2223

Faculdade de Ciências Humanas, Exatas e da Saúde do Piauí, IESVAP, Brasil

E-mail: Lahuan.araujo.costa@gmail.com

Gabriela de Souza Mendonça

ORCID: https://orcid.org/0000-0002-7922-5995

Faculdade de Ciências Humanas, Exatas e da Saúde do Piauí, IESVAP, Brasil

E-mail: gabyaraujo2007@ hotmail.com

Ligia Viana de Araújo

ORCID: https://orcid.org/0000-0002-0674-4604

Faculdade de Ciências Humanas, Exatas e da Saúde do Piauí, IESVAP, Brasil

E-mail: ligiaviana00@gmail.com

Vitória Stefanny Cunha Araújo

ORCID: https://orcid.org/0000-0002-3901-2207

Faculdade de Ciências Humanas, Exatas e da Saúde do Piauí, IESVAP, Brasil

E-mail: Vitoriastefannycunhq@gmail.com

Ana Maria Santos Cardoso

ORCID: https://orcid.org/0000-0001-7092-2295

Faculdade de Ciências Humanas, Exatas e da Saúde do Piauí, IESVAP, Brasil

E-mail: anamariacardoso05@gmail.com

Paulo Victor de Sousa Jordão

ORCID: https://orcid.org/0000-0001-9227-6159

Faculdade de Ciências Humanas, Exatas e da Saúde do Piauí, IESVAP, Brasil

E-mail: paulojordaopj@ hotmail.com

Brenda Dias Araujo

ORCID: https://orcid.org/0000-0001-5140-0934

Faculdade de Ciências Humanas, Exatas e da Saúde do Piauí, IESVAP, Brasil

E-mail: brenda150600@gmail.com

Charles Ponte de Sousa Filho

ORCID: https://orcid.org/0000-0002-0943-410X

Faculdade de Ciências Humanas, Exatas e da Saúde do Piauí, IESVAP, Brasil

E-mail: Charlesponte23@gmail.com

Louise Ribeiro Teixeira

ORCID: https://orcid.org/0000-0001-7378-1096

Faculdade de Ciências Humanas, Exatas e da Saúde do Piauí, IESVAP, Brasil

E-mail: ribeiroteixeiralouise@gmail.com

José Guilherme de Oliveira Rodrigues Ferreira

ORCID: https://orcid.org/0000-0002-2617-5146

Faculdade de Ciências Humanas, Exatas e da Saúde do Piauí, IESVAP, Brasil

E-mail: jgorf13@gmail.com

Rafaela Costa Pacheco

ORCID: https://orcid.org/0000-0001-8477-8298

Faculdade de Ciências Humanas, Exatas e da Saúde do Piauí, IESVAP, Brasil E-mail: pachecoc.rafaela@gmail.com

André Pessoa Silva Bastos

ORCID: https://orcid.org/0000-0002-1188-0766

Faculdade de Ciências Humanas, Exatas e da Saúde do Piauí, IESVAP, Brasil

E-mail: Andrepsbastos@gmail.com 
Brenda Ellen Menezes Cardoso

ORCID: https://orcid.org/0000-0002-5982-5985

Faculdade de Ciências Humanas, Exatas e da Saúde do Piauí, IESVAP, Brasil

E-mail: cbrendaellen@hotmail.com

Larruama Soares Figueiredo de Araújo

ORCID: https://orcid.org/0000-0002-0902-9200

Faculdade de Ciências Humanas, Exatas e da Saúde do Piauí, IESVAP, Brasil

E-mail: larru_pp@hotmail.com

Bianca Sampaio Lima

ORCID: https://orcid.org/0000-0002-0574-4936

Faculdade de Ciências Humanas, Exatas e da Saúde do Piauí, IESVAP, Brasil

E-mail: biancasp1234@gmail.com

Vivian Saeger Pires

ORCID: https://orcid.org/0000-0002-8852-0363

Faculdade de Ciências Humanas, Exatas e da Saúde do Piauí, IESVAP, Brasil E-mail: Viviansaegerpires@gmail.com

Artur Frota Guimarães

ORCID: https://orcid.org/0000-0003-1265-1671

Faculdade de Ciências Humanas, Exatas e da Saúde do Piauí, IESVAP, Brasil

E-mail: arturfrotaguimaraes@hotmail.com

Luan Kelves Miranda de Souza

ORCID: https://orcid.org/0000-0002-8019-4022

Faculdade de Ciências Humanas, Exatas e da Saúde do Piauí, IESVAP, Brasil Universidade Federal do Piauí, Brasil

E-mail: luan.souza@iesvap.edu.br

\title{
Resumo
}

A pandemia de coronavírus afetou de forma extensa as mais diversas camadas da sociedade, especialmente o setor acadêmico, causando assim, um déficit nas atividades de ensino pesquisa e extensão. Diante disso, criou-se a necessidade de desenvolvimento e aplicação de novas ferramentas, utilizando as Tecnologias de Informação e Conhecimento (TICs) e metodologias ativas, criando um ambiente equilibrado e estimulante, propício para o desenvolvimento de novas habilidades e reconfigurando o processo de ensino-aprendizagem. Destarte, esse estudo trata-se de um relato de experiência sobre o projeto "Caixa Mental" como um exemplo de ferramenta inovadora que objetiva promover as atividades de ensino e extensão universitária no período da pandemia incitada pela COVID-19. Esse projeto foi operante durante seis meses e consistiu na produção de uma série disponibilizada em oito capítulos que abordou as neurociências por meio de temas da teoria da mente, sendo composto por uma etapa de planejamento e uma etapa de execução. O projeto possuiu uma abordagem pautada nas metodologias ativas e TICs dentro do novo fazer extensionista. Nesse sentido, as novas tecnologias de informação e comunicação foram utilizadas como ferramentas pedagógicas inovadoras, construindo um saber científico de forma dinâmica e interativa. Posto isso, o projeto apresenta-se de grande valia, podendo esse modelo ser adotado para a difusão de conhecimentos em outros campos acadêmicos.

Palavras-chave: Extensão; Ensino-aprendizagem; Inovação; TICs; COVID-19.

\begin{abstract}
The coronavirus pandemic has extensively affected the most diverse stratums of society, especially the academic sector, causing a deficit in teaching, research and extension activities. Therefore, there was a need to develop and apply new tools, using Information and Knowledge Technologies (ICTs) and active methodologies, creating a path to the development of new skills and reconfiguring the teaching-learning process. Thus, this study is an experience report of the "Mind box" project as an example of an innovative tool that aims to promote teaching and university extension activities during the pandemic period incited by COVID-19. This project was active for six months and consisted of the production of a serie available in eight chapters that approached the neurosciences through themes of the theory of mind, consisting of a planning stage and an execution stage. The project had an approach based on active methodologies and ICTs in the new way to do extension. In this sense, the new information and communication technologies were used as innovative pedagogical tools, building scientific knowledge in a dynamic and interactive way. Hence, this model of project can be adopted for the dissemination of knowledge in other academic fields.
\end{abstract}

Keywords: Extension; Teaching-learning; Innovation; ICTs; COVID-19.

\section{Resumen}

La pandemia de coronavirus ha afectado ampliamente a los más diversos estratos de la sociedad, especialmente al sector académico, provocando un déficit en las actividades de docencia, investigación y extensión. Por tanto, existía la necesidad de desarrollar y aplicar nuevas herramientas, utilizando las Tecnologías de la Información y el Conocimiento (TIC) y metodologías activas, creando un entorno equilibrado y estimulante, propicio para el desarrollo de nuevas habilidades y reconfigurando el proceso de educación. Así, este estudio es un relato de experiencia del proyecto "Caja Mental" como ejemplo de una herramienta innovadora que tiene como objetivo promover la actividad docente y de extensión universitaria durante el período pandémico incitado por COVID-19. Este proyecto estuvo 
activo durante seis meses y consistió en la producción de una serie dispuesta en ocho capítulos que abordó las neurociencias a través de temas de la teoría de la mente, consistente en una etapa de planificación y una etapa de ejecución. El proyecto contó con un enfoque basado en metodologías activas y TIC dentro del nuevo proceso de extensión, en este sentido, las nuevas tecnologías de la información y la comunicación fueron utilizadas como herramientas pedagógicas innovadoras, construyendo conocimiento científico de forma dinámica e interactiva. Por tanto, el proyecto es de gran valor y este modelo puede ser adoptado para la difusión del conocimiento en otros campos académicos.

Palabras clave: Extensión; Enseñanza-aprendizaje; Innovación; TIC; COVID-19.

\section{Introdução}

A pandemia de coronavírus afetou de forma extensa as mais diversas camadas da sociedade, desde pequenos comerciantes a grandes multinacionais, sendo que uma das áreas mais atingidas foi a acadêmica. Devido às mais diversas medidas sanitárias, que possuíam como um de seus princípios primordiais o isolamento social, as aulas presenciais na grande maioria das universidades do Brasil e do mundo foram suspensas, assim como as atividades de pesquisa e de extensão, causando um enorme déficit, até a migração, muitas vezes demorada, para os sistemas remotos, passando inclusive pelo abandono repentino de projetos (DeFilippis, et al, 2020).

Dados da UNESCO estimam que mais de $90 \%$ dos estudantes do mundo tiveram suas aulas interrompidas por conta das medidas restritivas, com efeito, os corpos discente, docente e administrativo tiveram que se adaptar, no intuito de reduzir os danos pedagógicos e os riscos à saúde, ao mesmo tempo que precisaram manter o interesse e compromisso com o aprendizado. Diante disso, criou-se a necessidade de desenvolvimento e aplicação de novas ferramentas que mantivessem o interesse dos estudantes e o bom nível do conhecimento repassado simultaneamente, criando um ambiente equilibrado e estimulante, propício para o desenvolvimento de novas habilidades intelectuais e sócio afetivas, reconfigurando o processo de ensino-aprendizagem (Gatti, et al, 2020).

Dessa forma, a educação online está em ascensão durante o atual contexto, gerando uma crescente constante do número de alunos à distância (Abdulkader e Santos, 2020). Além disso, as instituições tomaram providências para informar, tranquilizar e manter o contato com os alunos e pais. Por conseguinte, é evidente que esta emergência na saúde mundial não configura um cenário para colocar em prática planos institucionais complexos e tradicionais para o ensino (Daniel, 2020), devendo-se incorporar estratégias de aprendizagem remota em ambiente educacional, visando permitir uma experiência favorável de estudantes, mesmo em distanciamento social. Para os docentes, o primeiro desafio foi a aquisição de conhecimentos acerca das ferramentas digitais adotadas. Outra questão relevante foi a inadequação dos recursos utilizados, compostos geralmente por redes de internet e dispositivos pessoais (Santos e Ferreira, 2021).

Concomitantemente à transição para o ensino digital, instituições adeptas de métodos ativos de ensino, como o Problem Based Learning (PBL) e Team Based Learning (TBL), que rompem com o modelo tradicional de ensino e fundamentam-se em uma pedagogia problematizadora onde o aluno é estimulado a assumir uma postura ativa em seu processo de aprendizagem visando uma conduta ativa e autônoma, encontraram um desafio a mais durante a pandemia (Paiva, et al, 2016). A soma de todas essas adversidades demonstrou a necessidade de desenvolver métodos inovadores que permitam aliar os fundamentos do método ativo às novas ferramentas remotas, visando diminuir ao máximo os prejuízos causados pela suspensão de suas atividades em ambiente acadêmico.

Destarte, esse estudo trata-se de um trabalho qualitativo do tipo relato de experiência sobre o projeto "Caixa Mental" como um exemplo da utilização das tecnologias remotas em consonância com as metodologias ativas, como uma ferramenta inovadora com o fito de promover as atividades de ensino e extensão universitária no período da pandemia incitada pelo novo coronavírus-SARS-CoV-2 (COVID-19). 


\section{Metodologia}

Mormente, para a fundamentação teórica de todos os procedimentos a descritos adiante, foi realizado um levantamento a respeito de modalidades alternativas para a realização de projetos de extensão acadêmica, utilizando as bases de dados eletrônicas Scielo (Scientific Electronic Library Online), ABC Health Sciences e Google Acadêmico. Os descritores utilizados foram extensão, educação, pandemia e inovações, todos presentes nos Descritores em Ciências da Saúde (DeCS). De acordo com Pereira. et al. (2018), esse trabalho trata-se de um estudo com abordagem qualitativa do tipo pesquisa-ação.

O projeto intitulado "Caixa mental”, elaborado pela Liga Acadêmica de Neurociências - LANEC- FAHESP/IESVAP, do curso de medicina vinculada à Faculdade de Ciências Exatas, Humanas e da Saúde do Piauí/Instituto de Educação Superior do Vale do Parnaíba - FAHESP/IESVAP, localizada na cidade de Parnaíba-PI, foi operante durante seis meses e consistiu na produção de uma série disponibilizada em oito capítulos que abordou as neurociências por meio de temas da teoria da mente. O projeto teve como público alvo usuários da rede social Instagram e Youtube, com especificidade para estudantes de forma geral que tenham interesse por neurociências.

O projeto consistiu em duas etapas, uma etapa de planejamento e uma etapa de execução. Na etapa de planejamento foi realizada a seleção dos temas a serem levantados na série. A escolha dos temas foi realizada por meio de metodologias ativas, especialmente o brainstorming (tempestade de ideias) com os discentes e docentes envolvidos no projeto em reuniões que ocorriam semanalmente em regime de aprendizagem remota (REAR). O brainstorming incita a criatividade e o comportamento informacional, por esse motivo foi a principal ferramenta nessa etapa. Posto isso, foram escolhidos 8 temas da teoria da mente que possuem maior apreço popular e acadêmico, foram eles: memória, efeito placebo, meditação, sentimentos e emoções, realidade virtual, ansiedade, mecanismos da mentira e imagética motora. Após a seleção, foram organizadas capacitações com os estudantes para que se tornassem aptos a trabalhar o conteúdo para o grande público. As capacitações também ocorreram semanalmente em modo REAR.

A etapa de execução teve início com a elaboração de um protocolo de filmagem para que os acadêmicos fossem capazes de realizar as gravações de modo individual e padronizado, utilizando smartphones como ferramenta, dentro das normas de distanciamento social (Quadro 1). O projeto foi realizado de maneira remota, sendo que cada discente protagonizou um vídeo e também foram responsáveis pelo conteúdo e roteiro. Os demais acadêmicos envolvidos no projeto ficaram encarregados da edição e divulgação dos vídeos, bem como auxílio para o conteúdo. Com efeito, os acadêmicos realizaram as filmagens e encaminharam para a equipe de edição. A partir disso, foi produzido o conteúdo audiovisual da série (Imagem 1) e os vídeos puderam ser postados semanalmente (Imagem 2) sempre com uma divulgação prévia (Imagem 3). Os vídeos foram disponibilizados nas plataformas IGTV e YouTube vinculadas à liga acadêmica executora. A metodologia do projeto é mostrada de forma resumida e esquematizada na imagem 4.

Quadro 1- Instruções e procedimentos para as filmagens.

\section{Protocolo de filmagem para os vídeos da Caixa Mental}

\section{Roteiro}

- Elaborar um roteiro de texto direto, de modo a descrever todos os elementos que serão apresentados no vídeo;

- O início do vídeo deve conter uma introdução delimitando uma situação problema de acordo com o tema do vídeo;

- O conteúdo necessita ser explanado de modo claro e com linguagem predominantemente coloquial no desenvolvimento do vídeo, com o fim de atingir o maior público possível;

- O roteiro precisa responder aos seguintes questionamentos acerca do tema:

a) O que é? 

b) Quais são os exemplos disso?
c) Qual a aplicabilidade?
d) Qual o mecanismo neural promove isso?

- O vídeo deve ter uma conclusão objetiva e instigante, apresentando uma síntese e retomando pontos importantes do vídeo.

\section{Preparação do material}

- O vídeo deve ser gravado em filmadora ou smartphone com a resolução mínima de 720p;

- O áudio deve ser gravado separadamente em outro dispositivo, utilizando microfone de lapela ou fone de ouvido com microfone;

OBS: O áudio próprio da filmadora ou smartphone não deve ser usado.

- O local de filmagem deve ser claro (luz natural ou artificial branca) e silencioso o máximo possível;

- Utilizar exclusivamente o uniforme da liga ou camiseta branca simples como figurino.

\section{Gravação}

- $\quad$ O formato do vídeo deve ser horizontal, 16:9;

- O vídeo deve possuir entre 5 e 10 minutos, sendo os 10 a 15 segundos iniciais destinados para a introdução e os 20 segundos finais destinados para a conclusão;

- Certificar-se de retirar todos os elementos do cenário que podem atrair a atenção do expectador. O fundo do vídeo precisa ser o mais limpo e organizado possível, de preferência sólido e em cores neutras;

- A câmera deve permanecer fixa em um ponto durante toda a filmagem. Se possível, utilize um tripé;

OBS: Em hipótese alguma outra pessoa deve gravar o vídeo segurando a filmadora com as próprias mãos, isso resultará em oscilações indesejadas no vídeo.

- O enquadramento precisa seguir as seguintes delimitações:
a) Superior: aproximadamente um palmo acima da cabeça;
b) Inferior: imediatamente abaixo da linha infra mamilar.
c) Laterais: o cenário escolhido.

\section{Comunicação}

- Pareça natural no vídeo, para isso, evite decorar o roteiro, repasse o conteúdo com as suas palavras e de modo proporcional ao conhecimento adquirido;

- Gesticule no com as mãos e utilizando expressões faciais para aumentar o seu poder de convencimento, no entanto, não em demasia, pois isso causaria cansaço visual no telespectador;

- Evite repetir interjeições e expressões como "né", "e aí", "tipo", dentre outras interruptamente, isso provoca cansaço auditivo e sensação de falta de domínio do conteúdo no telespectador.

\section{Equipe e procedimentos}

- Em virtude da pandemia causada pelo causada pelo novo coronavírus-SARS-CoV-2 (COVID-19), o vídeo deve ser feito apenas pelo próprio apresentador, com o fim de evitar aglomerações;

- Higienizar as mãos com álcool em gel 70\% antes e após tocar na câmera ou outros objetos do cenário;

- Higienizar os materiais com álcool líquido $70 \%$ ao início e fim da gravação.

6. Envio

- O material deve ser enviado exclusivamente anexado a um drive online para o e-mail laneciesvap@gmail.com ;

- Não serão aceitos os materiais enviados por aplicativos de conversa multimídia ou redes sociais. 
Fonte: Sales, PCSLS.

O quadro acima tem o intuito de apresentar instruções para a filmagem de vídeos a indivíduos inexperientes de modo claro e objetivo. Cabe salientar o item 5 do quadro, que concerne à equipe e produção, no qual foram apresentadas medidas para controle e prevenção da COVID-19.

Imagem 1- Pôster oficial da série.
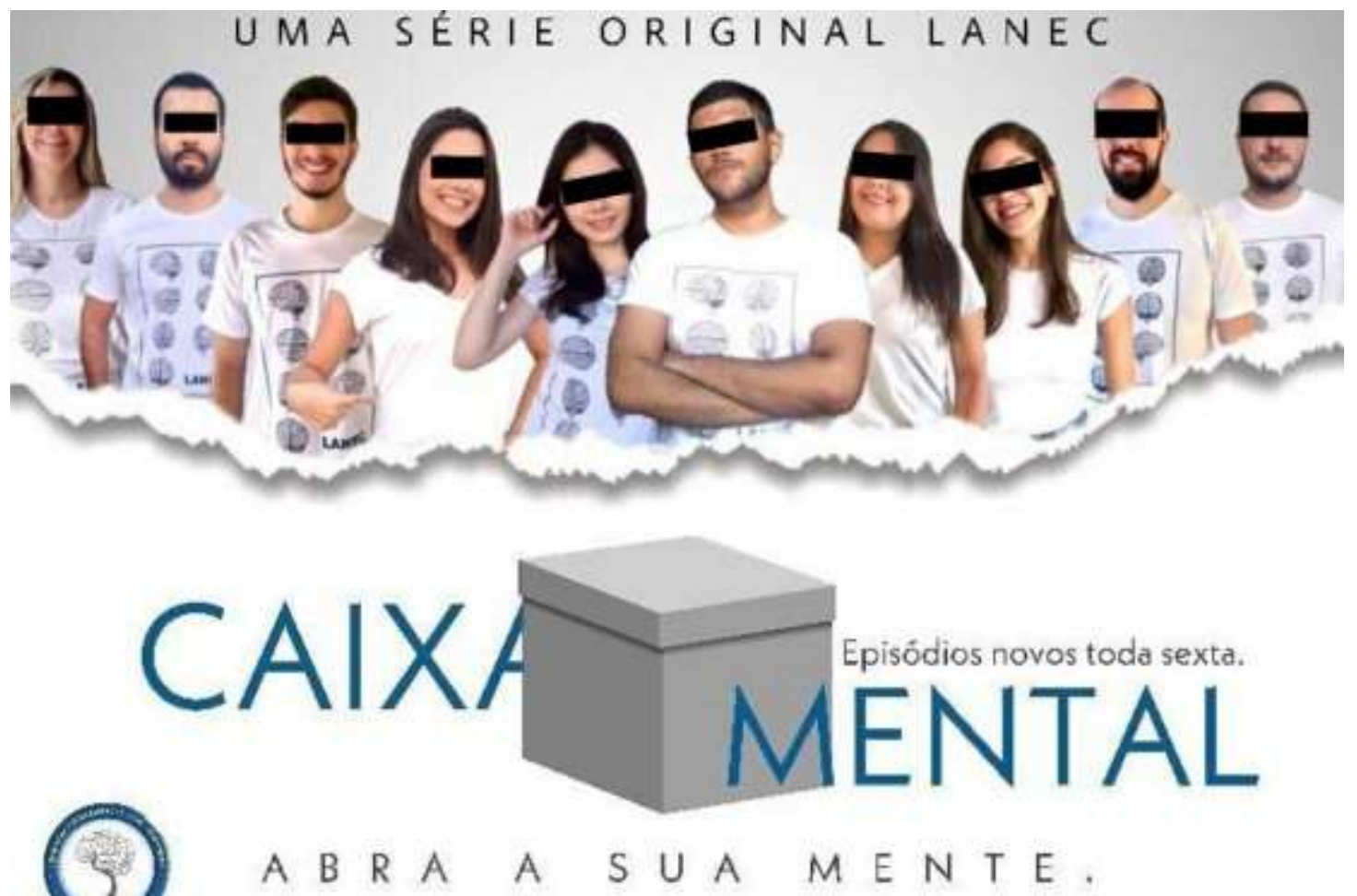

Fonte: Sales, PCSLS.

O pôster acima, utilizado para anunciar a série, tem o intuito de gerar interesse no público alvo. Cabe destacar que as informações verbais e não verbais foram utilizadas de modo a mimetizar os pôsteres de produções originais das plataformas de streaming, como Netflix e Amazon Prime Video, resgatando elementos da cultura pop. O pôster conta com todos os membros da equipe executora. 
Research, Society and Development, v. 10, n. 2, e51510212799, 2021

(CC BY 4.0) | ISSN 2525-3409 | DOI: http://dx.doi.org/10.33448/rsd-v10i2.12799

Imagem 2- Prints dos vídeos mostrando os acadêmicos protagonistas.
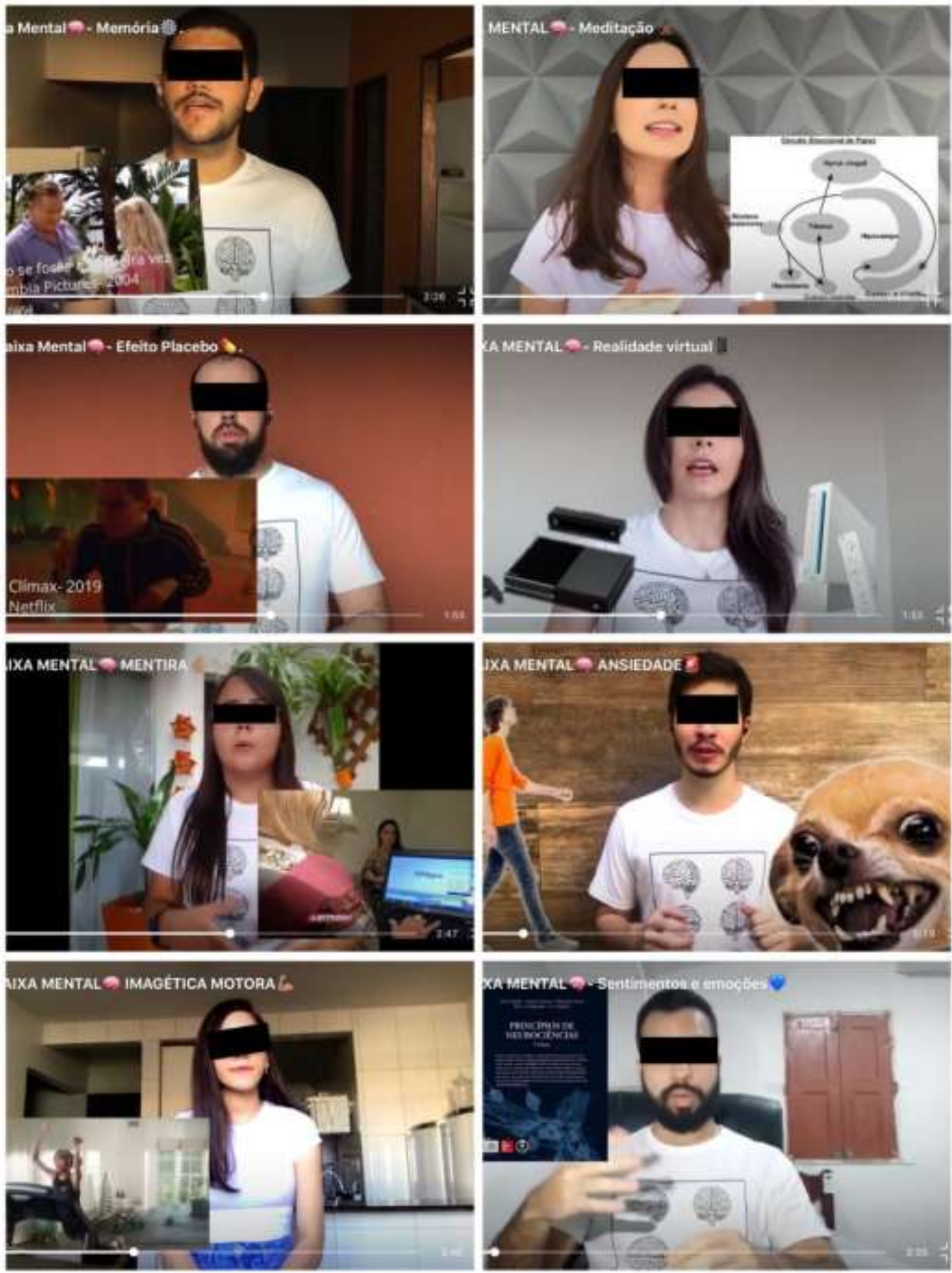

Fonte: Sales, PCSLS.

Os vídeos foram produzidos pelos participantes do projeto em suas próprias casas, como é possível observar na imagem supra inserida. Ademais, é possível notar que o protocolo mostrado no quadro 1 foi devidamente seguido pelos membros da equipe. Vale ressaltar ainda os elementos de edição de vídeo. 
Imagem 3- Pôsteres de divulgação individual dos vídeos.

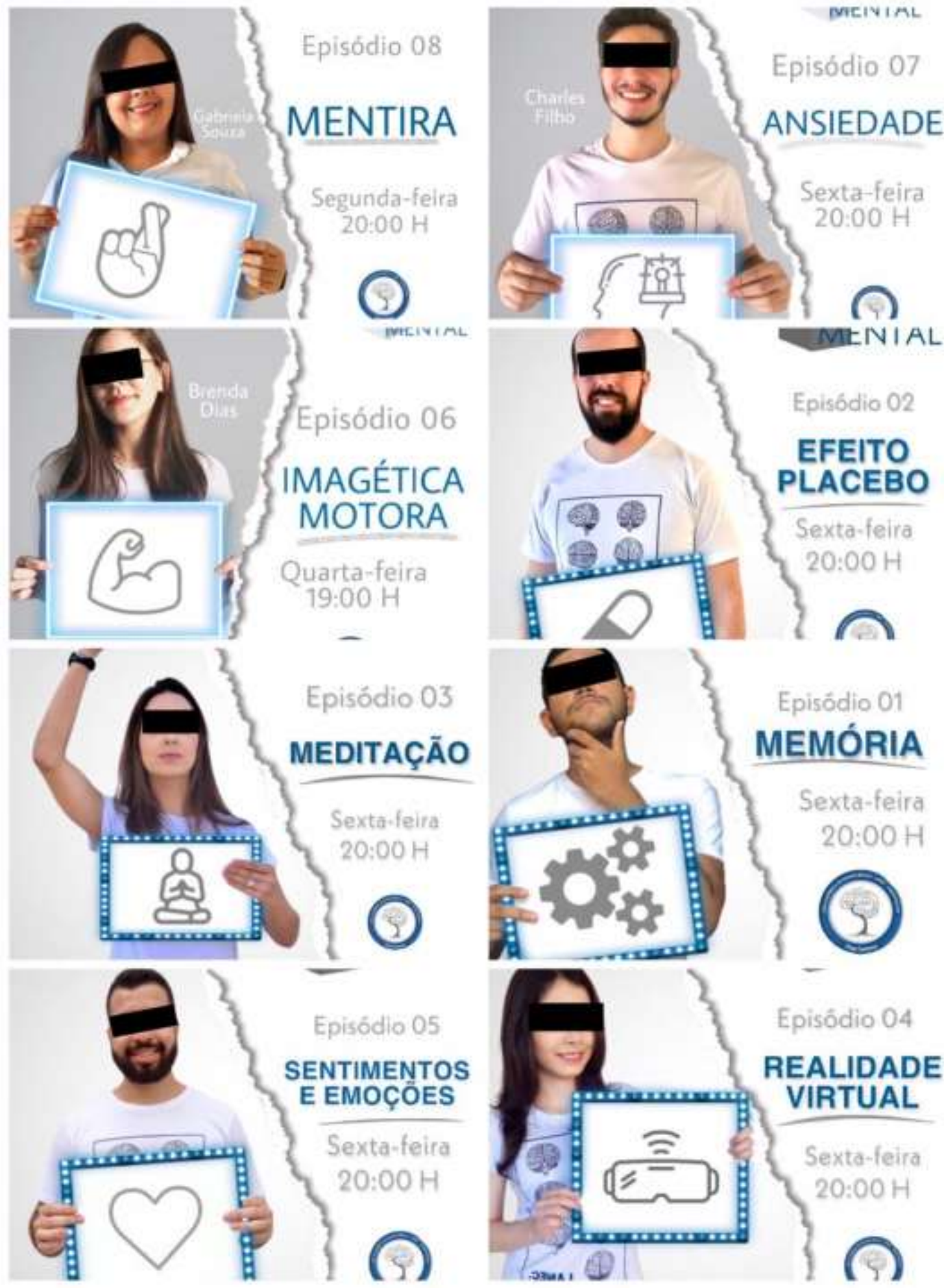

Fonte: Sales, PCSLS.

Os pôsteres individuais, mostrados superiormente, tiveram a função de apresentar de forma mais detalhada os apresentadores dos vídeos, trazendo familiaridade e criando uma proximidade com o público-alvo. Cabe acentuar ainda que cada tema foi associado a um ícone visual, mostrado nas placas que os apresentadores estão segurando, com o intuito de gerar uma memória visual e despertar ainda mais o interesse. 
Imagem 4- Fluxograma representando a metodologia do projeto "Caixa Mental”.

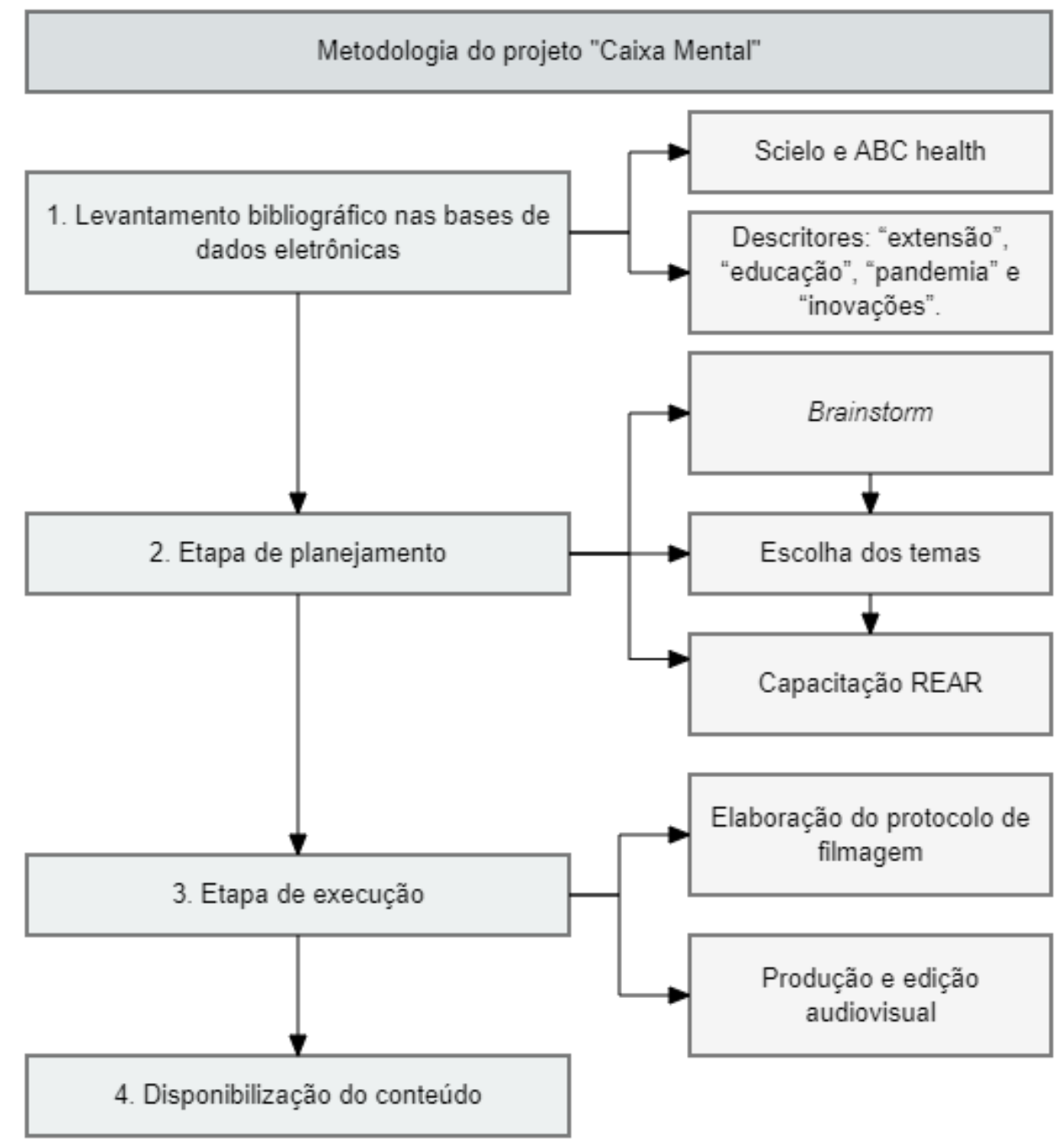

Fonte: Sales, PCSLS.

O esquema mostrado acima tem a função de apresentar de forma rápida e eficiente a metodologia do projeto, destacando as etapas separadamente (levantamento bibliográfico, planejamento, execução e disponibilização) e mostrando por um esquema de vetores (de cima para baixo) e ordem numérica (ordem crescente) a evolução e sequência dessas etapas.

\section{Resultados e Discussão}

O projeto "Caixa mental", uma série com oito episódios contemplando assuntos relacionados a teoria da mente, possuiu uma abordagem pautada nas metodologias ativas e TICs dentro do novo fazer extensionista. Dessa maneira, apresentou grande monta no que tange à apropriação de conhecimento dos destinatários, haja vista que por meio dele permitiuse o acesso a novas informações, com destaque para abordagem de assuntos atípicos do conhecimento acadêmico e popular. Nesse sentido, as novas tecnologias de informação e comunicação foram utilizadas como ferramentas pedagógicas inovadoras no projeto, com o objetivo de construir um saber científico de forma dinâmica e interativa com o público, utilizando uma linguagem apropriada a população.

A extensão universitária tem relevante papel na formação profissional, conectando a universidade à sociedade (Santos, et al, 2021). Essa troca de saberes permite que a extensão tenha três funções: a acadêmica, pautada por conhecimento 
teórico-metodológico; a social, permitindo a organização e a construção de cidadania; e a articuladora, através das ações (Cardoso, et al, 2021). Cabe salientar que a extensão universitária, como tantos outros setores da sociedade, foi bastante afetada pela pandemia, pois ações sociais que seriam realizadas em locais estabelecidos para seus públicos alvos, não puderam acontecer (Nunes, et al, 2021). Essa realidade social expõe e fragiliza os indivíduos, fazendo com que as relações entre educação e saúde passem a ser medidas por outros elementos, diferentes dos anteriores. Dessa forma, houve a necessidade de reinventar o fazer extensionista visando manter o tripé universitário (de Melo, et al, 2020).

O momento atual de pandemia torna necessário que os indivíduos se dediquem, ainda que de longe, a proteger e acolher as pessoas em suas demandas, com atendimentos de qualidade; e muitos docentes mostraram perseverança e compromisso social nessa busca. Conciliar a vida privada com as atividades laborais acadêmicas no esquema de home-office vem sendo um desafio para todos e foi neste contexto que docentes mostraram habilidades e competências aliando criatividade, inquietude, proatividade e capacidade de mobilização e articulação para o desenvolvimento de atividades extensionistas adaptadas à nova realidade (Moutinho, 2021).

Nesse sentido, as Tecnologias da Informação e Comunicação (TICs) invadiram todos os espaços da sociedade e, assim sendo, também atingiram o meio acadêmico. Com efeito, a nova era na pandemia está marcada por uma era da informatização e de conhecimento, diante disso, vários modelos pedagógicos tem se apropriado dos recursos digitais, em que estratégias síncronas e assíncronas estão a serviço do desenvolvimento de processos educacionais que viabilizem práticas intervencionistas de construção do conhecimento (Barboza, et al, 2020). Na Educação Superior, as TICs contribuem em um primeiro momento como repositório e ambiente de compartilhamento de atividades e material de apoio, e em segundo momento para a oferta de educação a distância, bem para como atividades de pesquisa e extensão durante o panorama hodierno (Conceição e Moreira, 2020).

Além disso, a presença das TICs implicou em uma mudança social e cultural e tem valorizado um novo tipo de conhecimento, que exige o domínio de habilidades no campo das ciências, (Briccia e Veloso, 2021) com os usuários em torno das diversas ferramentas tecnológicas, como computadores, televisores digitais e celulares, com conectividade à internet, logrando acesso em milhares de plataformas e aplicativos digitais. Entre os principais meios utilizados, estão: Zoom, Hangouts, Skype, YouTube, RNP, Sistemas Integrados, E-mail, Whatsapp, Facebook e Instagram (Soares e Colares, 2020).

Sob essa óptica, os dispositivos digitais são ferramentas que auxiliam os sujeitos da aprendizagem no processo de interpretação e de análise, principalmente no regime de ensino remoto. Por meio de sua tecnologia avançada, a internet faz um convite ao estabelecimento de uma nova relação com o saber. A partir do conhecimento proposto, os estudantes acabam desenvolvendo competências no sentido de aprender a construir o conhecimento, por meio de atividades e instrumentos científicos que sejam realmente significativos (Boell e de Arruda, 2021). Posto isso, a contribuição deste estudo está em responder as demandas de extensão universitária afetadas pela COVID-19 de uma forma clara e eficaz, abrindo lacunas para a replicação do modelo também em outras áreas.

\section{Considerações Finais}

Destarte, o projeto "Caixa Mental” traz à luz a descoberta de inovações pedagógicas e produção de material tecnológico no panorama hodierno da COVID-19, utilizando as TICs em consonância com as metodologias ativas. Em razão da série ter um público alvo inteiramente composto por usuários de plataformas digitais de comunicação, considera-se uma ferramenta de longo alcance, podendo esse modelo ser adotado para a difusão de conhecimentos além das neurociências, se expandindo para outros campos acadêmicos. Espera-se, portanto, que a partir desse estudo possam ser abertos caminhos para que temáticas de outras esferas do saber sejam exploradas, utilizando e reformulando essas ferramentas pedagógicas em trabalhos futuros. 
Research, Society and Development, v. 10, n. 2, e51510212799, 2021

(CC BY 4.0) | ISSN 2525-3409 | DOI: http://dx.doi.org/10.33448/rsd-v10i2.12799

\section{Referências}

Barboza, L. E. M., Silveira, I. S. D., Leite, P. C., Christoffel, M. M., Gomes, A. L. M., Souza, T. V. D., \& Silva, G. C. L. D. (2020). Os conceitos de Florence Nightingale em tempos de pandemia da COVID-19 retratados em história em quadrinhos: relato de experiência. Escola Anna Nery, 24(SPE).

Boell, M., \& de Arruda, A. A. (2021). Narrativas docentes e discentes no ensino superior: ensino remoto emergencial em tempos de pandemia da Covid-19 e a relação com a cultura digital. Brazilian Journal of Development, 7(1), 9963-9977.

Cardoso, M. C., Ferreira, C. P., da Silva, C. M., de Melo Medeiros, G., Pacheco, G., \& Vargas, R. M. (2021). Utilização das redes sociais em projeto de extensão universitária em saúde durante a pandemia de COVID-19. Expressa Extensão, 26(1), 551-558.

Cordeiro, K. M. D. A. (2020). O Impacto da Pandemia na Educação: A Utilização da Tecnologia como Ferramenta de Ensino. IDAAM.

Conceição, M. G., \& Rocha, U. R. (2020). TIC para manutenção dos estudos em período de pandemia na Universidade Federal da Bahia. Folha De Rosto, 6(2), 95-106

DeFilippis, E. M., Stefanescu Schmidt, A. C., \& Reza, N. (2020). Adapting the educational environment for cardiovascular fellows-in-training during the COVID-19 pandemic. J Am Coll Cardiol, 75 (20) 2630-2634.

Diniz, E. G. M., da Silva, A. M., Nunes, P. H. V., Franca, W. W. M., da Rocha, J. V. R., da Silva, D. V. S. P., ... \& de Lima Aires, A. (2020). A extensão universitária frente ao isolamento social imposto pela COVID-19. Brazilian Journal of Development, 6(9), 72999-73010.

Gatti, B. A. (2020). Possível reconfiguração dos modelos educacionais pós-pandemia. Estudos Avançados, 34(100), 29-41.

Melo, J. A. C., da Silva, C., de Souza Alves, M. L., de Souza Machado, I., Laurindo, M., \& Fin, A. P. C. (2020). Extensão universitária na pandemia de COVID-19: projeto radiologia na comunidade, o uso da rede social e ambiente virtual de aprendizagem. Saberes Plurais: Educação na Saúde, 4(2), 49-60.

Moutinho, F. F. B. (2021). Extensão universitária: uma luz na escuridão da pandemia de covid-19. Intermedius-Revista de Extensão da UNIFIMES, 1(1), 6372 .

Nunes, R. K. S., dos Santos Maciel, G. A., Almeida, E. B., Guedes, M. R., \& Henn, R. (2021). Desafios e adaptações da extensão universitária em tempos de pandemia: relato de experiência. Revista Ciência Plural, 7(1), 211-223.

Paiva, M. R. F., Parente, J. R. F., Brandão, I. R., \& Queiroz, A. H. B. (2016). Metodologias ativas de ensino-aprendizagem: revisão integrativa. SANARERevista de Políticas Públicas, 15(2).

Pereira, A. S., Shitsuka, D. M., Parreira, F. J., \& Shitsuka, R. (2018). Metodologia da pesquisa científica.

Santos, A. S. F., Martins, É. F., Sarmento, T. S., De Oliveira, J. L., Rodrigues, C. A. Q., \& Pinto, M. D. Q. C. (2021). Banco de instrumental odontológico: extensão universitária durante o período de pandemia da COVID-19. Expressa Extensão, 26(1), 514-521.

Santos, J. R.., Ferreira, M. E. (2021). Um relato de ensino de Química no contexto da pandemia de COVID-19 na rede pública de São Paulo: O desafio das aulas virtuais na Educação Básica. Research, Society and Development, 10 (2) 8710212267.

Vasconcelos Soares, L., \& Colares, M. L. I. S. (2020). Educação e tecnologias em tempos de pandemia no Brasil. Debates em Educação, $12(28)$, $19-41$.

Veloso, A. M. F., Briccia, V. (2021). O professor remoto: a reinvenção da prática pela fluência digital. Research, Society and Development, 10 (2) 3810212138 . 